\title{
Evaluating the Potential Impact of Pharmacogenomics on ADRs
}

\author{
EYAL OREN, BA; GARY MCCART, PharmD; AND KATHRYN A. PHILLIPS, PhD
}

I n this issue, Zachry et al. ${ }^{1}$ report on a survey of health care professionals' perceptions of the impact of pharmacogenomic information. Pharmacogenomics is the study of the interaction of an individual's genetic makeup and response to a drug. Choosing therapy based on likely drug response, identifying patients who will respond to a given medication, and identifying those persons at high risk for adverse drug events are some of the important issues that respondents in the survey perceived pharmacogenomic information should address. Survey respondents also perceived that the effect of pharmacogenomic information on physician preference for choosing a drug therapy was "high impact."

We agree with the findings from this survey that the role of pharmacogenomics in drug response is an area of great clinical, social, and economic importance. Specifically, in this review, we examine the potential impact of pharmacogenomics in reducing adverse drug reactions (ADRs) and on drug response. ADRs, which in 1994 were ranked between the fourth and sixth leading cause of death in the United States, are estimated to affect more than 2 million hospitalized patients in the United States alone ${ }^{2}$ and to occur most often during treatment with usual doses of FDAapproved drugs.

A growing number of articles have enumerated potential roles for pharmacogenomics in reducing ADRs. ${ }^{3-6}$ Pharmacokinetic considerations such as drug absorption, distribution, metabolism, and excretion and pharmacodynamic parameters such as receptor response are all highly variable due to multiple factors, including disease, environment, and genetics. Increased or decreased metabolism of a drug can change the systemic concentrations of the drug and its metabolites. Genetic polymorphisms, gene variations occurring in more than $1 \%$ of a population, may thus explain why a certain proportion of a population may not respond or be at higher risk for drug toxicity ${ }^{6}$ and are at greater risk for unexpected side effects.

In this subject review, we describe a set of criteria that can be used to evaluate the impact pharmacogenomic information may

\section{Authors}

EYAL OREN, BA, is Research Associate, Department of Clinical Pharmacy, School of Pharmacy, University of California, San Francisco; GARY MCCART, PharmD, is Professor of Clinical Pharmacy, Department of Clinical Pharmacy, School of Pharmacy, University of California, San Francisco; KATHRYN A. PHILLIPS, PhD, is Associate Professor of Health Economics and Health Services Research, Department of Clinical Pharmacy, School of Pharmacy and Institute for Health Policy Studies, University of California, San Francisco.

AUTHOR CORRESPONCE: Gary McCart, PharmD, Professor of Clinical Pharmacy, Department of Clinical Pharmacy, School of Pharmacy, University of California, San Francisco, 521 Parnassus Ave., Box 0622, San Francisco, CA 94143. Tel.: (415) 476-1733; Fax: (415) 502-0792; E-mail: gmccart@itsa.ucsf.edu have on ADRs and drug response., ${ }^{3,7}$ These criteria could serve as a foundation to develop more comprehensive cost analyses of pharmacogenomic data as well as serve as future starting points in assessing clinical and societal implications. These criteria are presented in Table 1.

By providing specific examples of drugs that illustrate these criteria, we also intend to demonstrate aspects in which the decisionmaking process of a provider or prescriber could be affected by knowing the genetic information of the patient. For each example, we have reviewed the role of the drugs in therapy, the genetic deficiency and clinical effect(s), and the medical significance and treatment options. Choosing a drug therapy based on genetic information could significantly alter the course of therapy and the associated outcomes

\section{Current Level of Medical Need}

- Prevalence of adverse drug reactions and the use of the associated drug are high enough to warrant use of genetic intervention.

Drugs affected by the MDRl/p-glycoprotein polymorphism provide a good illustration of this criterion as they are commonly used and associated with a potential high level of adverse drug reactions that could possibly be prevented.

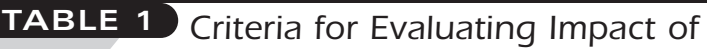 Pharmacogenomic Information}

Current level of medical need:

a) Prevalence of adverse drug reactions and the use of the associated drug are high enough relative to each case to warrant use of genetic information.

b) The prevalence of abnormal metabolizers of a given drug due to variant alleles is high.

c) The outcome of administering the wrong drug or dose of a drug is severe enough to result in high mortality, significant changes in quality of life, or lead to expensive medical costs

d) Current methods of monitoring drug response or evaluating toxic effects are ineffective or not available.

Clinical utility of using genetic information. The information is likely to have greater clinical utility if a strong association exists between the genetic variant and clinical response and eventual patient outcome to a drug.

Feasibility of use of the information. The information is more likely to be used if a relatively cheap, quick, and reliable genetic test can detect the variant allele using genotyping and if clinicians are then able to interpret results and use the information appropriately. 


\section{Role of the Drugs in Therapy}

Drug transporter proteins are normal membrane proteins that play important roles in the normal function of cells. They are also known to be important in the therapeutic use of drugs because, based on gene expression, they promote or limit drug uptake in tissues such as the large and small intestine, liver, kidney (proximal tubules), and brain (capillaries). Drug transporter proteins include organic anion and cations, lung resistance protein, p-glycoprotein, and other multidrug resistance proteins (normal cell membrane proteins that transport a variety of drug substrates and influence drug effect by limiting drug distribution and response).

\section{Genetic Deficiency and Clinical Effect}

Polymorphisms in the multidrug-resistance gene (MDR1) produce variations in p-glycoprotein expression that change the body concentration of a wide range of drugs that are substrates for them. There are more than 15 polymorphisms of MDR1 producing variations in p-glycoprotein. P-glycoprotein transports xenobiotics, drugs, and their metabolites out of normal cells and cancer cells, thus "protecting" them.

One MDR1 polymorphism (Exon 26, T allele) that transports protease inhibitors (PI) also is known to be associated with higher plasma digoxin levels. This polymorphism is more common in European Americans (62\%) and African Americans (13\%). ${ }^{9}$ Hoffmeyer et al. noted a 24\% incidence of the homozygous variant (Exon 26, $\mathrm{T}$ allele) for decreased p-glycoprotein expression in his study $(\mathrm{n}=188) .{ }^{10}$ Hoffmeyer also noted a $38 \%$ increase in steady-state digoxin levels in a small sample $(\mathrm{n}=14)$ of healthy volunteers homozygous for the polymorphism for decreased p-glycoprotein activity. Although the Hoffmeyer data should be relevant to PIs (indinavir, nelfinavir, saquinavir) Fellay et al. noted unexpected low plasma levels of the PIs nelfinavir and efavirenz in patients with the same polymorphism (Exon 26, T allele). In the Caucasian (European) population studied, the homozygous (TT) incidence of the polymorphism was $25 \%$. It is possible that other transporters and metabolizing enzymes were somehow compensating for the expected higher plasma levels. ${ }^{11}$

\section{Medical Significance and Treatment Options}

P-glycoprotein affects the transportation of many commonly used drugs; digoxin, cyclosporine, vinca alkaloids, HIV protease inhibitors (PI), and dexamethasone are typical drug substrates. Many of these drugs have relatively low therapeutic indexes, and, thus, small increases in serum or tissue levels will affect toxicity. MDR1 polymorphisms with decreased expression of p-glycoprotein may increase PI (saquinavir, indinavir, nelfinavir, ritonavir, and amprenavir) levels and cause an increased incidence of toxicity, which may include insulin resistance, glucose intolerance, and hyperlipidemia. ${ }^{12,13}$ These and other PI toxicities may be related to decreased p-glycoprotein expression and related CYP4503A4/5 metabolizing activity. ${ }^{14}, 15$ Since PI toxicity often limits AIDS therapy, a better understanding of the genetic basis of PI use may increase therapeutic options for the treatment of AIDS. For example, this might affect the current use of Highly Active AntiRetroviral Therapy (HAART) that suppresses HIV replication, delays the onset of AIDS, and prolongs patient survival.

\section{- The prevalence of abnormal metabolizers of a given drug due to variant alleles is high.}

Isoniazid and its association with the N-Acetyltransferase-2 (NAT2) polymorphism provide an illustration of this criterion, as slow acetylators of NAT2 are highly prevalent in certain population groups.

\section{Role of the Drugs in Therapy}

Isoniazid is one of the primary drugs for the treatment and prevention of tuberculosis. The slow acetylation of isoniazid and its relationship to peripheral neuropathy is a well-studied and classic example of a genetic variation in drug metabolism that causes an adverse drug reaction. ${ }^{16}$ In addition, fast acetylators of isoniazid have higher levels of toxic metabolites that are associated with hepatitis. ${ }^{17} \mathrm{~N}$-acetyltransferase (NAT2) is a hepatic and extrahepatic enzyme responsible for the metabolism (acetylation) of several drugs including isoniazid, procainamide, hydralazine, and a variety of sulfa drugs. Furthermore, isoniazid is a substrate for other drugmetabolizing enzymes (CYP2C9 and 2C19) that metabolize drugs like phenytoin. Thus, slow metabolizers of isoniazid are also at risk for phenytoin toxicity from increased drug levels. ${ }^{18}$

\section{Genetic Deficiency and Clinical Effect}

Acetylation is considered an inactivating pathway that conjugates drug substrates to water-soluble moieties with enhanced elimination. Polymorphisms of NAT2 alter the incidence of adverse effects and perhaps the efficacy of acetylated drugs. Prevalence of poor metabolizers in certain groups is particularly high. For example, slow acetylators include 91\% of Egyptians, 50\%-59\% of Caucasians (both European and North American), 41\% of African Americans, 20\% of Chinese, and 8\%-10\% of Japanese. Three to five alleles of NAT2 account for 90\% of the slow acetylation metabolizing enzymes. Fast acetylators metabolize procainamide to renally excreted and therapeutically active N-acetyl-procainamide (NAPA). Thus, fast acetylators with renal dysfunction accumulate NAPA and are at increased risk for adverse drug reactions. Slow acetylators of hydralazine and perhaps procainamide are prone to develop a lupus-like syndrome.

\section{Medical Significance and Treatment Options}

Because the clinical impact of NAT2 polymorphisms have been well known and appreciated since the 1950s, genotyping or phenotyping is generally not used. For example, pyridoxine (Vitamin B6) can be routinely given to patients taking isoniazid to prevent peripheral neuropathy. This appears to be cost-effective prevention. The clinical safety of daily isoniazid is also typically managed with routine 
liver monitoring to prevent liver damage, but in the case of fast acetylators, it might not be cost-effective or optimal care; the prevalence criteria should also be viewed in terms of severity.

- The outcomes of administering the wrong drug or dose is severe enough to result in high mortality or significant changes in quality of life or lead to expensive medical costs.

Warfarin and its association with the drug-metabolizing enzyme CYP2C9 provide an example of severe outcomes when therapy is not given at the appropriate dose.

\section{Role of the Drugs in Therapy}

Warfarin is an anticoagulant widely used for the prevention and treatment of thromboembolic disorders. There is a narrow therapeutic dosing range for warfarin, and careful monitoring is required to maintain an appropriate balance between efficacy and serious bleeding events. Factors such as diet, drug use, and certain disease states may affect this balance.

\section{Genetic Deficiency and Clinical Effect}

Warfarin is a racemic mixture of r-warfarin and s-warfarin, and different enzymes metabolize each racemic form. The hepatic microsomal enzyme CYP2C9 is primarily responsible for the metabolism of s-warfarin. S-warfarin is 3 times more potent than r-warfarin. Two common polymorphisms of CYP2C9 (CYP2C9*2 and CYP2C9*3) exist with a frequency of $11 \%$ and $7 \%$, respectively, in Caucasians (North Americans). Both polymorphisms have significantly decreased ability (30\% and $80 \%$, respectively) to metabolize s-warfarin. ${ }^{19}$ Recently, Higashi et al. noted in a retrospective cohort study that patients taking warfarin with at least one CYP2C9 polymorphism had an increased risk of an international normalized ratio higher than the target INR and took more time to establish stable dosing. ${ }^{19}$ More importantly, these patients had a significantly increased risk of a serious bleeding event. The authors recommended caution in interpreting these results due to the small numbers of patients involved. It should also be noted that this study included a homogeneous population (those of Asian and African descent were excluded), and other potentially important metabolizing and blood-clotting polymorphisms were not evaluated. Patients were not excluded due to diet, drug use, or other medical conditions.

\section{Medical Significance and Treatment Options}

This study by Higashi et al. points to the potential for pharmacogenetics. Testing for polymorphisms of CYP2C9 may improve warfarin dosing and prevent serious bleeding events. Testing for CYP2C9 polymorphism is available from Genelex Corporation (www.genelex.com). Larger and more comprehensive prospective studies are required to confirm the clinical role of CYP2C9 testing in the use of warfarin.
- Current methods of monitoring drug response or evaluating toxic effects are ineffective or not available.

HMG-CoA reductase inhibitors (statins) and their association with the cholesteryl ester transfer protein (CETP) gene polymorphism provide an example of a pharmacogenomic intervention that may be more effective than traditional monitoring methods.

\section{Role of the Drugs in Therapy}

HMG-CoA reductase inhibitors improve the survival of patients with coronary artery disease, the leading cause of death in the United States. A variety of polymorphisms may affect cholesterol levels and perhaps the effect of statins on the progression of coronary atherosclerosis in patients with coronary artery disease.

\section{Genetic Deficiency and Clinical Effect}

A cholesteryl ester transfer protein (CETP) gene polymorphism (taqIB) creates increased levels of CETP. CETP plays a key role in the metabolism of high-density lipoprotein (HDL) as it uses HDL and other lipoproteins to transfer cholesterol to the liver, where cholesterol exits into the bile. High levels of HDL are associated with reduced risk of coronary artery disease. The high plasma levels of CETP and reduced levels of HDL found with the taqIB polymorphism are thought to be strong risk factors for atherosclerosis as a part of a complex risk system.

Kuivenhoven et al., in a 2-year study, compared the effect of pravastatin versus placebo on atherosclerosis regression in 807 Dutch men with coronary atherosclerosis. ${ }^{20}$ The primary outcome was the reduction in coronary artery diameter with and without the presence of the CETP polymorphism (taqIB or B1). Each treatment group was divided into 3 categories based on those (1) who were homozygous for the polymorphisms (B1B1) or (2) heterozygous (B1B2) for the polymorphism, and those (3) without the polymorphism (B2B2). In both the pravastatin and placebo control groups, those patients with the polymorphism had the highest CETP levels and the lowest HDL levels. In the placebo group, the mean decrease in coronary artery lumenal diameter (indicating disease progression) was greater in the homozygous polymorphism category (B1B1). This reduction was not seen in the pravastatin BlBl group. However, in the homozygous-dominant B2B2 category of the pravastatin group ( $16 \%$ of the population), pravastatin did not halt the lumen reduction (disease progression). In the pravastatin group, after 2 years, improvements in total cholesterol, LDL, HDL, and triglycerides were improved and not significantly different in any of the categories (B1B1, B1B2, or B2B2). However, pravastatin significantly reduced coronary artery narrowing in the B1B1 and somewhat in the B1B2 category but not for the patient category without the polymorphism (B2B2). The authors concluded that the presence of the polymorphism (B1B1, B1B2) and the increased levels of CETP might predict the disease response to pravastatin. 


\section{Medical Significance and Treatment Options}

If these data are valid, there is a group of patients without the polymorphism and that potentially has progressive coronary artery atherosclerosis, that appears to be responding based on improved lipid levels. In this case, however, lipid levels, which would ordinarily be used for monitoring, do not adequately predict response.

There are many important limitations to this research, including the significance and usefulness of the outcome measure, other effects of statins, environmental factors, diet, interpretation of cholesterol risk factors, and conflicting results from other studies. Studies that measure myocardial infarction, coronary artery bypass surgery, percutaneous coronary angioplasty, and death will be needed to confirm these data. Other studies have linked fluvastatin and simvastatin effectiveness with other gene polymorphisms. ${ }^{21}$

These studies and the provocation they provide, however, are good examples of the conceptual changes in therapy we can expect to see as we learn more about the genetic basis of disease and drug use.

\section{Clinical Utility of Using Genetic Information}

The information is likely to have greater clinical utility if a strong association exists between the genetic variant and clinical response and eventual patient outcome to a drug. In other words, high gene penetrance (a high correlation between the presence of a gene variation and clinical response to a drug) should exist, and a surrogate marker of drug toxicity or outcome will be tightly linked to each variant allele. The assay should also be predictive for a substantial portion of the patient population.

Mercaptopurine/azathioprine/thiopurine and the TPMT deficiency provide an example of high gene penetrance and a successful assay that has been developed.

\section{Role of the Drugs in Therapy}

The enzyme thiopurine methyl transferase (TPMT) catalyzes the conjugation of the methyl group in the anti-leukemic agents 6-mercaptopurine and 6-thioguanine and in azathioprine, an immunosuppressive used to prevent rejection in transplant surgery and to treat autoimmune disease.

\section{Genetic Deficiency and Clinical Effect}

Several of the TPMT alleles containing gene-inactivating mutations have been identified. Three mutant alleles, TPMT*2, TPMT*3A, and TPMT*3C, account for the majority of the mutant alleles. Inheritance of these alleles has been shown to be responsible for the failure of drug therapy in the treatment of childhood leukemia (ALL), where giving the regular dose of thiopurine drugs may even result in bone marrow toxicity that may be fatal. Other adverse reactions include pancreatitis, infection, and hepatitis. One in 300 children is born with no activity for TPMT, and 10\% are born without full activity for the enzyme. The dosage needed in patients without full TPMT activity can require up to a 15 -fold reduction of the standard dose. ${ }^{22}$

\section{Medical Significance and Treatment Options}

This makes TPMT a great candidate for DNA-based genotyping tests that can screen susceptible patient populations before drug treatment begins. Through an agreement with St. Jude Children's Research Hospital, where the mutations that cause toxicity were discovered, 5 commercial tests for the mutations are now available throughout Europe and the United States (www.DNA.com), allowing physicians to determine which children with leukemia must take adjusted dosages. The test classifies patients according to their level of TPMT activity. Interest is now emerging in using TPMT genotyping for transplant patients, patients with Crohn's disease, systemic lupus erythematosus, and other autoimmune diseases. ${ }^{23}$

\section{Feasibility of Use of the Information}

The information is more likely to be used if a relatively cheap, quick, and reliable genetic test can detect the variant allele and if clinicians are then able to interpret results and use the information appropriately. A strategy to maximize sensitivity and specificity of tests in predicting phenotypes is essential. Moreover, since the information derived from genetic testing to predict drug response likely could be used throughout the patient's lifetime, a more expensive immediate test cost could be offset by other potential uses of the genetic information.

Trastuzumab (Herceptin) and its binding to the human epidermal growth factor receptor-type 2 (HER2 receptor) illustrates the successful use of a genetically based therapy.

\section{Role of the Drugs in Therapy}

Trastuzumab is an anti-HER2 monoclonal antibody that binds to the HER2 receptor and inhibits the transmission of several growth signals. Treatment with trastuzumab in stage IV breast cancer patients, who have received previous chemotherapy and who have tested positive for the over-expression of this protein biomarker, has produced cancer regression in at least $15 \%$ to $20 \%$ of patients. In addition, it has been found that trastuzumab also increases the efficacy (30\% to $45 \%$ ) of other breast cancer chemotherapies (e.g., paclitaxel [Taxol]) for patients who have not had previous chemotherapy. ${ }^{24}$

\section{Genetic Deficiency and Clinical Effect}

Genetic research has helped clinicians to better understand the cause, progression, and spread of cancer. It has also been important in the development of new drug therapies, especially for the treatment of breast cancer. For example, the HER2 proto-oncogene encodes a cell receptor protein that is overexpressed on the surface of tumor cells of $25 \%$ to $35 \%$ of patients with primary breast cancer. These patients tend to have more aggressive disease and shortened survival than those who do not overexpress HER2..$^{25}$ Several 
growth factors exert cancer growth stimulatory effects on the HER2 cell surface receptors, and, thus, blocking the HER2 receptor should result in a suppression of further tumor growth.

\section{Medical Significance and Treatment Options}

In 1998, the FDA approved trastuzumab for "metastatic breast cancer patients whose tumors overexpress the HER2 protein and who have received one or more chemotherapy regimes for their metastatic disease." Herceptin (in combination with paclitaxel) is also indicated for treatment of these same patients who have not received chemotherapy. These uses, however, require specific testing and documentation of HER2 overexpression. ${ }^{26}$

Initial clinical trials with trastuzumab were done using immunohistochemistry (IHC) that measures HER2 overexpression. Immunohistochemistry testing is FDA-approved and marketed as Dako Hercep Test and the Ventana Medical Systems' Pathway HER2/neu. Some of the advantages of IHC testing are that the testing is widespread, inexpensive (about $\$ 100$ per test), has more familiar technology, rapid throughput, and requires minimal technologist time. Disadvantages include the need for rigid controls, possible false positives, and subjective interpretations. Results are reported on a 0-3 scale where 0 and 1 indicate normal expression and 3 is a positive for overexpression. ${ }^{27}$

Fluorescence in-situ hybridization (FISH) testing such as Abbott/Nysis PathVysion is also FDA-approved and measures HER2 gene amplification in the nucleus. Advantages of FISH testing include flexible testing conditions and objective interpretation, while the disadvantages include more complex technology, interpretation time, and expense (about $\$ 400$ per test). Results are reported as either positive or negative for overexpression. The choice of the testing method is controversial and complicated by the many variables that make direct comparison of tests difficult.

While this testing improves cancer regression probability, other genetic factors as well as age, diet, environment, other gene amplification/overexpression, and other regulatory enzymes may play an important role in improving the selection of patients for trastuzumab treatment. Ongoing studies will determine if trastuzumab will be beneficial to patients with early stage (I-III) breast cancer. Herceptin will likely be used with other antineoplastic agents and in other cancers where HER2 is overexpressed.

At this time, commercially available genetic tests are still few and far-between. In addition to tests for HER2 and TPMT, CYP450 tests are also under development. But while testing is slowly making its way into commercial use, it is proving to be expensive. A recent check of the Genelex Corporation's Web site mentioned earlier (Redmond, WA) ${ }^{28}$, where CYP450 tests are being offered, showed pricing for a single gene test at $\$ 135-\$ 155$ (not including the lab fee). The goal of making a test fast and simple (as is the case for known point mutations) can also be difficult, as not all of the deficiencies can be attributed to the most common mutations. Analyzing the complete gene can also be a time-consuming and expensive undertaking. ${ }^{29}$

\section{Conclusion}

Pharmacogenomics is ushering in a new era for drug treatment through the application of individualized therapy based on underlying genetic variability. Careful evaluations are needed on a caseby-case basis before investing resources in research and development of pharmacogenomic-based therapeutics. There is much work yet to be done, all the way from identifying and categorizing polymorphisms for specific alleles to developing routine screening methods that can be used to predict response to specific drugs. The survey results provided by Zachry and Armstrong illustrate the growing need for understanding pharmacogenomic information, in context. It is our hope that the criteria and examples presented above will aid clinicians' understanding of some of the underlying issues related to drug response, including the identification of patient populations appropriate for specific medication administration and prospective identification of the risk of adverse drug reactions. As our awareness of genetic variability expands, it is likely that newfound knowledge and therapies will successfully prevent adverse drug reactions and improve drug efficacy.

\section{REFERENCES}

1. Zachry W, Armstrong E. Health care professionals' perceptions of the role of pharmacogenomic data. J Man Care Pharm. 2002;8(4):278-84

2. Lazarou J, Pomeranz B, Corey P. Incidence of adverse drug reactions in hospitalized patients: A meta-analysis of prospective studies. JAMA. 1998;279(15):1200-05.

3. Phillips K, Veenstra D, Oren E, Lee J, Sadee W. Potential role of pharmacogenomics in reducing adverse drug reactions: A systematic review. JAMA. 2001;286(18):2270-79.

4. Roses A. Pharmacogenetics and the practice of medicine. Nature. 2000;405:857-65.

5. Evans W, Relling M. Pharmacogenomics: translating functional genomics into rational therapeutics. Science. October 15, 1999;286:487-91.

6. Meyer U. Pharmacogenetics and adverse drug reactions. Lancet. 2000;356:1671-71.

7. Veenstra D, Higashi M, Phillips K. Assessing the cost-effectiveness of pharmacogenomics. Pharm Sci. September 14, 2000;2(3, article 29). Available at: www.pharmsci.org.

8. Spear B. Viewpoint-Pharmacogenomics: today, tomorrow, and beyond Drug Ben Trends. 1999;11(2):53-54.

9. Kim R, Leake B, Choo E, et al. Identification of functionally variant MDR1 alleles among European Americans and African Americans. Clin Pharmacol Ther. 2001;70(189-99).

10. Hoffmeyer S, Burk O, von Richter O, et al. Functional polymorphisms of the human multidrug-resistance gene: multiple sequence variations and correlation of one allele with P-glycoprotein expression and activity in vivo. Proc Natl Acad Sci USA. March 28, 2000;97(7):3473-78

11. Fellay J, Marzolini C, Meaden E, et al. Response to antiretroviral treatment in HIV-1-infected individuals with allelic variants of the multidrug resistance transporter 1: a pharmacogenetics study. Lancet. 2002;359(9300):30-36

12. Murata H, Hruz P, Mueckler M. The mechanism of insulin resistance caused by HIV protease inhibitor therapy. J Biol Chem. 2000;275:20251-54.

13. Mulligan K, Grunfeld C, Tai V, et al. Hyperlipidemia and insulin resistance are induced by protease inhibitors independent of changes in body composition in patients with HIV infection. J Acquir Immune Defic Syn. 2000;23:35-43. 


\section{Evaluating the Potential Impact of Pharmacogenomics on ADRs}

14. Barry M, Mulcahy F, Merry C, Gibbons S, Back D. Pharmacokinetics and potential interactions amongst antiretroviral agents used to treat patients with HIV infection. Clin Pharmacokinet. 1999;36:289-304.

15. Maheny C, Lamb M, Brouwer K, Pollack G. Pharmacokinetic and pharmacodynamic implications of p-glycoprotein modulation. Pharmacotherapy. 2001;21:778-96.

16. Lunde PK, Frislid K, Hansteen V. Disease and acetylation polymorphism. Clin Pharmacokinet. May-June 1977;2(3):182-97.

17. Mitchell J, Thorgeirsson U, Black M, Timbrell J, Snodgrass W, Potter W. Increased incidence of isoniazid hepatitis in rapid acetylators: possible relation to hydrazine metabolites. Clin Pharmacol Ther. 1975;18:70-79.

18. Miller R, Porter J, Greenblatt D. Clinical importance of the interaction of phenytoin and isoniazid: a report from the Boston Collaborative Drug Surveillance Program. Chest. 1979;75:356-58

19. Higashi M, Veenstra D, Kondo L, et al. Association between CYP2C9 genetic variants and anticoagulation-related outcomes during warfarin therapy. JAMA. 2002;287(13):1690-99.

20. Kuivenhoven J, Jukema J, Zwinderman A, et al. The role of a common variant of the cholesteryl ester transfer protein gene in the progression of coronary atherosclerosis. N Eng J Med. 1998;338:86-93.

21. Humma L, Lam Y. Pharmacogenetics. In: Dipiro JT, Talbert RL, Yee GC, Matzke GR, Wells BG, and Posey LM, ed. Pharmacotherapy: A Pathophysiologic Approach. 5th ed. New York, NY: McGraw-Hill; 2002:55-68.
22. Iyer L, Ratain M. Pharmacogenetics and cancer chemotherapy. European J Cancer. 1998;34(10):1493-99.

23. Krynetski E, Evans W. Genetic polymorphism of thiopurine s-methyltransferase: molecular mechanisms and clinical importance. Pharmacology. 2000;61:136-46.

24. Baselga J, Norton L, Albanell J, et al. Recombinant humanized anti-HER2 antibody (Herceptin) enhances the antitumor activity of paclitaxel and doxorubicin against HER2/neu overexpressing human breast cancer xenografts. Cancer Res. 1998;58:2825-31.

25. Hortobagyi G. Developments in chemotherapy of breast cancer. Cancer. 2000;88:3073-79

26. I. Herceptin [package insert]. San Francisco: Genentech Inc.; 2001

27. Check W. Making the call in HER2 testing methods. CAP TODAY. February 2002. Available at: www.cap.org. Accessed May 31, 2002.

28. Genelex. Available at: www.healthanddna.com/drugreactiontest.html. Accessed May 13, 2002

29. Krynetski E, Evans W. Pharmacogenetics as a molecular basis for individualized drug therapy: the thiopurine S-methyltransferase. Pharm Res. 1999;16(3):342-49

Hortobagyi G. Developments in chemotherapy of breast cancer. Cancer. 2000;88:3073-79. 